\title{
Diabetes, bone and glucose-lowering agents: basic biology
}

\author{
Beata Lecka-Czernik ${ }^{1,2,3}$
}

Received: 5 January 2017 / Accepted: 7 March 2017 / Published online: 22 April 2017

(C) The Author(s) 2017. This article is an open access publication

\begin{abstract}
Skeletal fragility often accompanies diabetes and does not appear to correlate with low bone mass or trauma severity in individuals with diabetes. Instead (and in contrast to those with osteoporotic bone disease), bone remodelling and bone turnover are compromised in both type 1 and type 2 diabetes, contributing to defective bone material quality. This review is one of a pair discussing the relationship between diabetes, bone and glucose-lowering agents; an accompanying review is provided in this issue of Diabetologia by Ann Schwartz (DOI: 10.1007/s00125-017-4283-6). This review presents basic science evidence that, alongside other organs, bone is affected in diabetes via impairments in glucose metabolism, toxic effects of glucose oxidative derivatives (advance glycation end-products [AGEs]), and via impairments in bone microvascular function and muscle endocrine function. The cellular and molecular basis for the effects of diabetes on bone are discussed, as is the impact of diabetes on the stem cell niche and fracture healing. Furthermore, the safety of clinically approved glucoselowering therapies and the possibility of developing a single therapy that would be beneficial for both insulin sensitisation and diabetes bone syndrome are outlined.
\end{abstract}

Electronic supplementary material The online version of this article (doi:10.1007/s00125-017-4269-4) contains a slideset of the figures for download, which is available to authorised users

Beata Lecka-Czernik

beata.leckaczernik@utoledo.edu

1 Departments of Orthopaedic Surgery, MS 1008, Health Sciences Campus, The University of Toledo, 3000 Arlington Avenue, Toledo, OH 43614, USA

2 Physiology and Pharmacology, Health Sciences Campus, The University of Toledo, Toledo, $\mathrm{OH}$, USA

3 Center for Diabetes and Endocrine Research, Health Sciences Campus, The University of Toledo, Toledo, OH, USA
Keywords AGEs · Bone quality · Bone remodelling · Bone vasculature $\cdot$ Glucose $\cdot$ Glucose-lowering therapies . Muscle $\cdot$ Osteoblast $\cdot$ Osteoclast $\cdot$ Review $\cdot$ Stem cells

$\begin{array}{ll}\text { Abbreviations } \\ \text { AGEs } & \text { Advanced glycation end-products } \\ \text { AMPK } & \text { AMP-activated protein kinase } \\ \text { BMD } & \text { Bone mineral density } \\ \text { DIO } & \text { Diet-induced obesity } \\ \text { DPP-4 } & \text { Dipeptidyl peptidase } 4 \\ \text { eNOS } & \text { Endothelial nitric oxide synthase } \\ \text { GLP-1 } & \text { Glucagon-like peptide 1 } \\ \text { HSCs } & \text { Haematopoietic stem cells } \\ \text { MSC } & \text { Mesenchymal stem cell } \\ \text { PPAR } & \text { Peroxisome proliferator activated receptor } \\ \text { RAGE } & \text { Receptor for advanced glycation end-products } \\ \text { RANKL } & \text { Receptor activator of nuclear factor } \mathrm{kB} \text { ligand } \\ \text { RUNX2 } & \text { Runt-related transcription factor } 2 \\ \text { SGLT2 } & \text { Sodium-glucose cotransporter } 2 \\ \text { TZDs } & \text { Thiazolidinediones } \\ \text { VSMC } & \text { Vascular smooth muscle cells }\end{array}$

\section{Introduction}

Skeletal fragility often accompanies type 1 and type 2 diabetes and is considered a pathological complication of this disease. Although low bone mass in type 1 diabetes may significantly contribute to an increase in fracture risk, an increase in fracture incidence is also observed in individuals with type 2 diabetes despite normal or even high bone mineral density (BMD) and greater BMI (factors that are considered protective against fractures in individuals without diabetes, as discussed further in an accompanying review by Ann Schwartz, in this issue of 
Diabetologia [1]). Similarly, fractures that often result from low or medium trauma cannot be explained by increased falls as a result of diabetes-related comorbidities [2]. Instead, despite high/normal BMD, in individuals with diabetes bone shows a number of structural characteristics predisposing it to fractures, including greater cortical porosity, smaller cortical area and decreased bone material strength [3, 4]. These features suggest that bone biomechanical quality is affected in individuals with diabetes.

Bone material quality is maintained by the process of bone remodelling, which is common for all mammals. Bone remodelling relies on constant resorption and rebuilding of bone in order to replace old tissue with new, more functional tissue. A balance between osteoclast-dependent bone resorption and bone formation, the latter of which relies on osteoblast activity, is essential for the maintenance of bone mass. Consequently, most metabolic bone diseases, including senile and postmenopausal osteoporosis, result from unbalanced bone remodelling. On the contrary, bone in diabetes is characterised by attenuated (rather than unbalanced) bone remodelling. Interestingly, decreased bone formation, as measured in iliac bone biopsies, was found to correlate with duration of diabetes [5] and circulating levels of biochemical markers of bone formation and resorption are shown to be decreased in diabetes [6]. It is speculated that low turnover of bone in diabetes may lead to defective microfracture repairs and, hence, to their accumulation, contributing to decreased bone quality. In contrast to postmenopausal and senile osteoporosis, a deterioration of bone strength in diabetes is associated with increased cortical porosity that is not accompanied by a loss of trabecular bone mass $[7,8]$. Thus, it can be concluded that diabetes-specific bone characteristics may constitute a novel syndrome that can be classified as a diabetes-associated bone disease. This review addresses the skeletal consequences of the major features that characterise diabetes, including impairments in glucose/insulin metabolism, accumulation of advanced glycation end-products (AGEs), insufficiency of the bone microvasculature and alterations in muscle endocrine function. Alongside the pleiotropic effects of these factors on bone degeneration, the impact of glycaemic control in response to existing glucose-lowering therapies is discussed; an overview of the clinical safety of glucose-lowering therapies is provided in the accompanying review [1], whereas this review focusses on the laboratory evidence of the effects of these therapies on bone.

\section{Insulin signalling, glucose metabolism and bone turnover}

Energy metabolism and bone turnover are controlled by intricate mechanisms that share many cues and outcomes. Because of the size of the skeleton and the extent of energy consumed during the process of bone remodelling, the skeleton is an organ of the body that heavily depends on glucose metabolism. Therefore, it is not surprising that insulin signalling plays an important role in the regulation of bone remodelling. More specifically, osteoblasts require glucose for differentiation and function [9], while glucose and insulin positively regulate the expression of runt-related transcription factor 2 (RUNX2) and bone-specific osteocalcin, the latter being a hormone that is implicated in the regulation of insulin sensitivity in peripheral tissues [10]. In addition, insulin increases support for osteoclastogenesis by decreasing the expression of osteoprotegerin, a decoy receptor for the pro-osteoclastic cytokine receptor activator of nuclear factor $\mathrm{kB}$ ligand (RANKL) [11]. Interestingly, the same system has recently been implicated in the regulation of insulin production in pancreatic beta cells, providing additional evidence that bone and energy metabolism are regulated by closely related mechanisms [12]. Taking into consideration that insulin signalling and glucose metabolism correlate positively with bone turnover and bone formation, a logical question arises as to whether bone develops insulin resistance and, if so, how it is manifested.

Some answers come from extensive studies in rodent models where mimicking insulin resistance in bone by depleting insulin receptor in cells of osteoblast lineage results in decreased bone formation and bone resorption and, as a consequence, decreased bone turnover $[11,13]$. In turn, manipulation of insulin signalling at the osteoblast level affects systemic energy metabolism. The postulated mechanism implicates bone turnover as a factor that controls energy balance through release of the bioactive form of osteocalcin from the mineralised matrix, which in turn stimulates insulin secretion [10]. Comparably, glucose intolerance is associated with the attenuation of bone remodelling and turnover in mouse models of diet-induced obesity (DIO) [14-16]. Interestingly, analogous with adult onset of type 2 diabetes, the development of DIO in adult mice that have achieved peak bone mass results in high bone mass and attenuated bone turnover [16]. The increased bone mass that accompanies DIO may reflect a protective mechanism, emulating the cessation of bone turnover that provides protection from bone loss that naturally occurs during ageing; however this mechanism does not seem to protect from the loss of bone material quality. Most importantly, osteoblasts in mice with DIO exhibit characteristics of insulin resistance as they do not respond to the stimulatory effect of insulin on the phosphorylation of IRS1/2. Saturated fatty acids and associated lipotoxicity appear to be culprits for the dysfunctional insulin signalling in bone from animals with DIO [14].

Molecular mechanisms linking bone homeostasis with glucose metabolism At the molecular level, skeletal homeostasis is linked to insulin sensitivity through the nuclear receptor peroxisome proliferator activated receptor (PPAR) $\gamma$. Most recently, it has been shown that the same post-translational 
modifications of the PPAR $\gamma$ protein that regulate insulin sensitivity and energy metabolism also regulate bone turnover, providing an insight into the intricate relationship between these processes [17]. PPAR $\gamma$ controls differentiation of cellular components of bone remodelling; it suppresses osteoblast differentiation by diverting marrow mesenchymal stem cell (MSC) commitment away from osteoblast and towards adipocyte lineage. Simultaneously, it also promotes recruitment of haematopoietic stem cells (HSCs), driving them towards the osteoclast lineage [18]. In MSCs, the activity of proadipocytic PPAR $\gamma$ and pro-osteoblastic RUNX2 is reciprocally regulated at the level of serine phosphorylation. Phosphorylation of S112 in PPAR $\gamma$ and S319 in RUNX2 is mediated by the same mitogen activated protein (MAP) kinases and results in activation of RUNX2 and inhibition of $\operatorname{PPAR} \gamma$, and subsequent differentiation of MSCs towards osteoblasts [19]. Conversely, activation of PPAR $\gamma$ and inhibition of RUNX2 activity requires activation of protein phosphatase 5 (PP5), resulting in dephosphorylation of S112 in PPAR $\gamma$ and S319 in RUNX2, and promoting MSC differentiation toward adipocytes, as opposed to osteoblasts [20]. With respect to bone-resorbing osteoclasts, PPAR $\gamma$ supports their differentiation through direct and indirect mechanisms. In monocytes, PPAR $\gamma$ stimulates osteoclast differentiation through peroxisome proliferator-activated receptor gamma, coactivator 1 , beta (PGC-1 $\beta$ )-dependent mechanism and activation of the c-Fos transcription factor, while, in MSCs, PPAR $\gamma$ increases support for osteoclastogenesis by stimulating RANKL production [21, 22]. Using selective modulators and genetic manipulation of PPAR $\gamma$ activity, it has been shown that dephosphorylation of S273, which determines the insulin sensitising activity of this protein, is required for PPAR $\gamma$ 's pro-osteoclastic activity, whereas phosphorylation of S112, which is known to prevent adipose tissue expansion, also correlates with increased bone formation [17]. Thus, these two PPAR $\gamma$ functions are necessary for balanced energy metabolism and insulin sensitivity, while also being inherently tied to processes regulating bone turnover through stimulation of bone formation and bone resorption. On the other hand, insulin resistance and obesity, which at the PPAR $\gamma$ level are associated with the phosphorylation of S273 and dephosphorylation of S112, correlate with decreased bone resorption and bone formation, providing a plausible explanation for attenuated bone turnover in type 2 diabetes.

The stem cell niche in diabetes Poor fracture healing and high infection rates constitute a significant clinical issue for individuals with diabetes. The factors discussed above may contribute to poor healing at the cellular level via their effect on the stem cell niche. Delta-like non-canonical Notch ligand 1 (DLK1) represents a common negative regulator of both skeletal stem cell differentiation and glucose metabolism via negative regulation of the osteocalcin-insulin loop [23].
Another strong indication of the link between skeletal stem cell differentiation and glucose metabolism has been provided by a recent study showing that low-grade inflammation in diabetes negatively affects the marrow stem cell niche, resulting in impaired fracture healing [24].

\section{The skeletal consequences of common diabetic characteristics}

AGEs and bone quality Type I collagen is a major constituent of bone and provides a structural framework that, upon mineralisation, facilitates the skeleton's strength. Type I collagen is a fibrillary protein that is organised around a triple-helix motif, causing self-assembly into highly organised fibrils stabilised by enzymatic cross-linking. Besides the natural enzymatic cross-linking, type I collagen may undergo chemical cross-linking, which can occur between 'free-floating' sugars in the serum and exposed amino acid residues, leading to posttranslational modifications of collagen and, as a result, the production of AGEs. Diabetes predisposes individuals to the accumulation of AGEs in many organs, including bone. With respect to structural properties, the accumulation of AGEs in bone and the formation of intra- and inter-cross-links in collagen fibres decrease bone biomechanical properties by increasing material stiffness. Studies on Zucker diabetic Sprague-Dawley (ZDSD) rats, a rodent model of type 2 diabetes, demonstrated that bone toughness significantly decreased with duration of diabetes despite normal mineralisation [25]. AGEs may also affect bone quality by activating signalling downstream of their receptor (the receptor for advanced glycation end-products [RAGE]). This signalling drives marrow MSC differentiation towards functional osteoblasts, promoting bone formation [26], and positively regulates HSC differentiation into osteoclasts, promoting bone resorption [27]. RAGE may also be produced as a soluble 'decoy' receptor that, by binding AGEs, may inhibit AGE-RAGE signalling axes. In type 2 diabetes, it has been shown that low serum levels of soluble RAGE and high serum levels of the AGE pentosidine are indicative of risk for fractures independent of BMD [28].

Bone vasculature in diabetes Bone vasculature is critical for bone growth, remodelling and injury healing. It provides a sustained supply of oxygen, nutrients and regulatory factors, and removal of metabolic waste. Up to $10 \%$ of cardiac output is distributed to the bone mineral compartment and bone marrow by a complex system of sinusoid and classic capillaries. It is conceivable that the same pathological changes that develop in diabetes in the peripheral vasculature also develop in bone. Thus, diabetic complications, including impairment in endothelium-dependent vasodilation, vascular calcification and defective angiogenesis, may affect the development of osteoblast progenitors from the haematopoietic niche 
and delivery of osteoblasts (pericytes) and osteoclasts to the bone remodelling unit by capillaries present in Harvesian canals [29]. The reduction in blood flow and impairment in new vessel formation may lead to a decrease in osteoblast formation, decrease in bone remodelling activity and, consequently, a decrease in bone quality and delayed fracture healing.

It has been recently reported that microvascular disease correlates with increased fractures in type 1 diabetes and deficits in the cortical bone in individuals with type 2 diabetes, as compared with individuals without microvascular disease [30, 31]. AGE/RAGE signalling heavily influences vascular calcification, as shown in a number of animal and human studies. Activation of RAGE by AGEs in vascular smooth muscle cells (VSMC) of the peripheral vascular system triggers a signalling cascade involving p38 mitogen activated protein kinase (MAPK), TGF- $\beta$ and NFkB. Subsequent downregulation of VSMC markers and activation of an osteoblast-like programme, including expression of Runx2 and osteocalcin and increased enzymatic activity of alkaline phosphatase, result in VSMC calcification [32]. Thus, it is possible that vessel calcification in bone may occur by the same mechanism and contribute to decreased blood flow and delivery of progenitors to the bone remodelling unit. Although there is a paucity of animal studies on blood flow and its role in the maintenance of bone homeostasis and impairments in diabetes, it has been demonstrated that the anabolic effect of intermittent parathyroid hormone (PTH) therapy is associated with increased blood flow in bone, suggesting a supportive role of microvasculature during bone formation [33].

\section{Muscle contribution to skeletal impairment in diabetes}

The interaction between bone and skeletal muscle occurs at a biomechanical and physiological level. Muscle action exposes bone to a variety of stimuli, including those generated in exercise. This stimulation can be conveyed in the form of direct force applied to the bone or in the form of released endocrine factors. In effect, both forms of muscle-generated signalling reach cellular components that regulate bone remodelling. Diabetes is often associated with limited regular exercise and a sedentary lifestyle, both of which contribute to metabolic impairment and systemic low-grade inflammation. During exercise, skeletal muscle produces myokines, which are released into the circulation and participate in the regulation of glucose and fatty acid metabolism in an autocrine and endocrine fashion. Among them, IL- 6 and irisin have been strongly implicated in coupling energy metabolism with bone metabolism.

The dual effect of the IL- 6 cytokine on glucose and bone metabolism has been well documented [34]. Metabolic impairment is accompanied by chronically increased levels of IL-6 in the circulation, which contribute to the development of insulin resistance. In contrast, IL-6 that is released from the muscle in response to exercise has an opposite effect, including reduction in systemic inflammation and increased glucose uptake by the muscle. In bone, IL- 6 has a comparable dual effect. Chronically increased levels of IL-6 increase RANKL production and osteoclastic bone resorption. However, mice deficient in IL-6 have low bone mass, reduced osteoblast number and delayed fracture healing [35] suggesting an important role for this cytokine in the maintenance of bone homeostasis. Most recently, it has been demonstrated that IL-6 production in murine exercising muscle is under the control of osteocalcin and that IL-6, through a feed-forward mechanism, increases circulating levels of bioactive osteocalcin [36].

Irisin, a myokine implicated in the 'beiging' of fat tissue and in improvements in insulin sensitivity, has been recently shown to have a positive effect on cortical bone [37]. Interestingly, this effect is autonomous in the bone and occurs independent of the effects of irisin on fat tissue metabolism. As demonstrated in vitro, irisin directly signals to MSCs through a specific, not as yet identified receptor and increases the expression of osteoblast gene markers. Together these studies provide a paradigm of the intricate mechanisms for reciprocal regulation of muscle, bone and energy metabolism. Moreover, they provide a plausible scenario for how impairments in energy metabolism may trigger a cascade of signalling events that may affect both bone and muscle.

Fig. 1 summarises the contribution of glucose metabolism, muscle, AGEs and vasculature impairment to decreased bone quality in diabetes.

\section{Evidence for the effect of glucose-lowering medications for bone health}

Glucose-lowering therapies target different aspects of glucose metabolism, including insulin sensitivity (metformin and thiazolidinediones [TZDs]), insulin secretion and bioactivity (sulfonylureas, glucagon-like peptide 1 [GLP-1] analogues, dipeptidyl peptidase 4 [DPP-4] inhibitors and insulin analogues), and modulation of blood glucose levels by either increased excretion (sodium-glucose cotransporter 2 [SGLT2] inhibitors) or delaying its appearance following nutrient digestion ( $\alpha$-glucosidase inhibitors and amylin [amylin is not currently approved for use in the UK]).

Therapies with either positive or neutral effects on bone The effects of metformin and sulfonylureas, which account for more than $70 \%$ of prescriptions for diabetes [38], are considered as neutral for human bone because there is no correlation between the use of these therapeutic agents and incidence of fractures. However, strong experimental evidence suggests that metformin may be beneficial for bone; in mesenchymal cells, metformin activates a pro-osteoblastic regulatory cascade via RUNX2 and AMP-activated protein kinase 


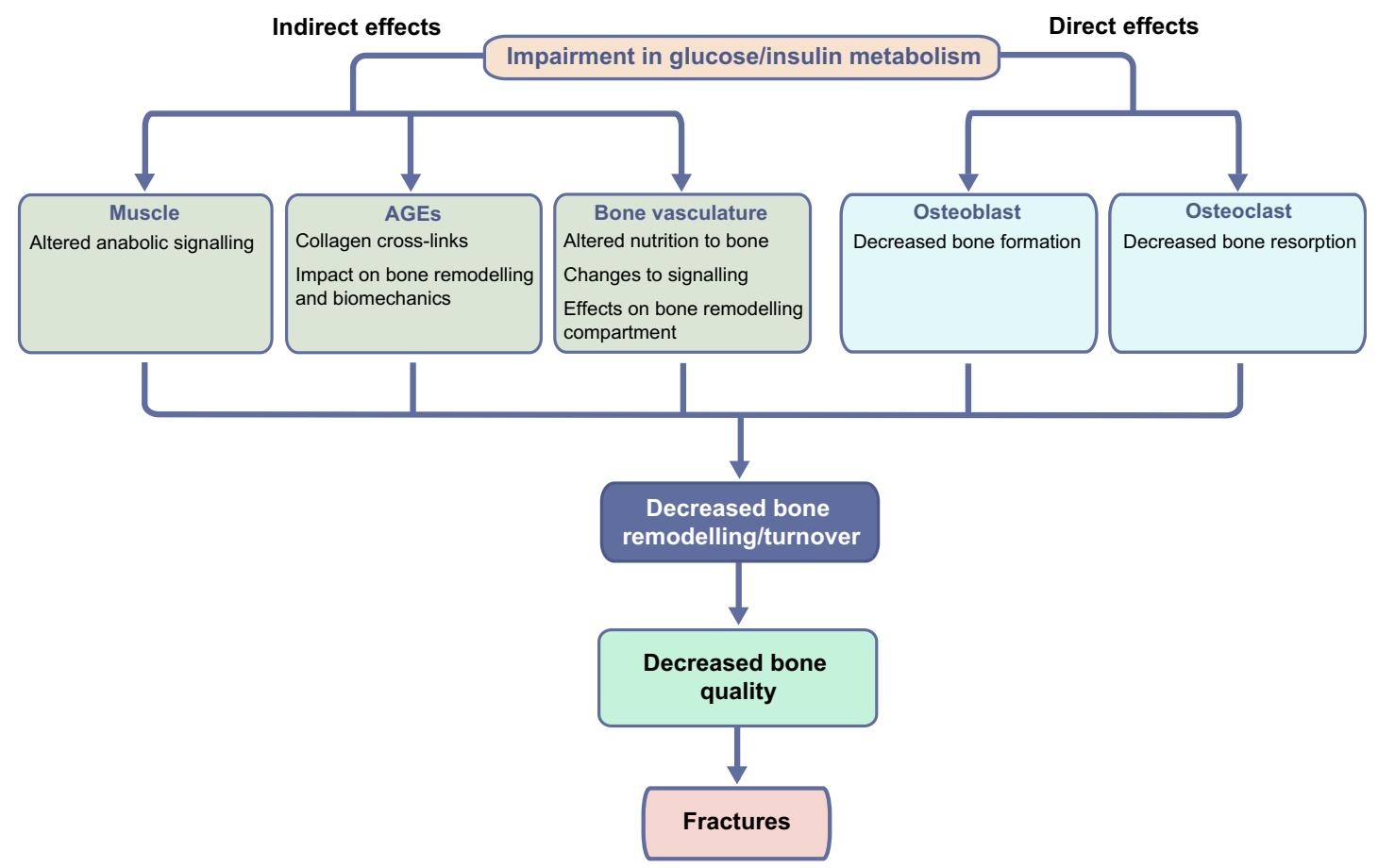

Fig. 1 Graphical summation of the effects of diabetes on bone. Impairments in glucose and insulin metabolism have indirect and direct effects on bone quality in individuals with diabetes. Impaired glucose/ insulin metabolism may indirectly affect bone by altering skeletal muscle signalling. Furthermore, in metabolic disease, the accumulation of AGEs may trigger pathways that promote collagen cross-linking (altering bone biomechanics) and impact on bone remodelling. Furthermore, the bone vasculature is disturbed with dysregulation in glucose and insulin metabolism, so that the delivery of nutrients and signalling factors

(AMPK)/upstream transcription factor 1 (USF1)/small heterodimer partner (SHP) signalling, and inhibits adipogenesis through both AMPK and mechanistic target of rapamycin (mTOR)/p70 S6 kinase $\left(\mathrm{p} 70^{\mathrm{S} 6 \mathrm{~K}}\right)$ signalling $[39,40]$. Further, in haematopoietic cells, metformin decreases osteoclast development and prevents macrophage proinflammatory responses to AGEs by decreasing RAGE signalling, potentially decreasing bone marrow support for resorption and proatherosclerotic effects on bone vasculature $[39,41]$. To this end, in mice with streptozotocin-induced type 1 diabetes, metformin improved the angiogenic functions of endothelial cells via activation of the AMPK/endothelial nitric oxide synthase (eNOS) pathway [42]. It has also been shown that metformin has a protective effect on bone mass in conditions of oestrogen deficiency [43]. Similarly, some research suggests that glimepiride, a second generation sulfonylurea, may enhance osteoblastic differentiation in high glucose conditions via the phosphoinositide 3-kinase (PI3K)/Akt/eNOS pathway [44] and bone formation in ovariectomised rats [45]. Experimental evidence may differ from clinical observations because the beneficial effects of metformin and sulfonylureas on human bone may be too subtle to be detected in the existing clinical trials, which were not designed to study the effects of these drugs on bone. (e.g. that regulate vasodilation) to the bone is impaired. The changes in bone vasculature in diabetes results in decreased remodelling activity in the bone. Impairments in glucose and insulin metabolism also directly impact on osteoblast and osteoclast activity, resulting in decreased bone formation and bone resorption. Ultimately, the indirect and direct effects of impaired glucose/insulin metabolism on bone lead to decreased bone remodelling/turnover, decreased bone quality and increased risk of fractures

Similarly, the skeletal effects of incretin-based therapies, such as GLP-1 analogues, in humans are not clear despite several in vitro and in vivo studies suggesting their beneficial effect. Both osteoblasts and osteoclasts express GLP-1 receptors and treatment of normoglycaemic ovariectomised mice with the receptor agonists, exenatide and liraglutide, increased trabecular, but not cortical bone mass through a combined effect on osteoblast and osteoclast activity [46].

Animal studies on the effect of DPP-4 inhibitors on bone have not shown consistent results.

Therapies with negative effects on bone SGLT2 inhibitors form a new class of glucose-lowering medications that have recently been scrutinised for their skeletal effects. There is a paucity of basic research studies on the effect of SGLT2 inhibitors on bone, however one study showed that canagliflozin may exacerbate trabecular bone loss in a streptozotocin-induced murine model of type 1 diabetes [47]. The effects may include increased bone resorption; however the exact mechanism for this outcome has not been demonstrated, raising a question as to whether canagliflozin affects bone directly or systemically [47].

The most notorious drugs that show a negative effect on bone are TZDs, which act as high affinity ligands and 
activators of PPAR $\gamma$. It is well documented that clinically approved TZDs, rosiglitazone and pioglitazone, decrease bone mass and increase incidence of fractures, especially in women. As full PPAR $\gamma$ agonists, dephosphorylating both S273 and S112 in the PPAR $\gamma$ protein, TZDs activate proadipocytic pathways and suppress pro-osteoblastic programmes in MSCs, while activating pro-osteoclastic programmes in HSCs both directly and via increased RANKL production in mesenchymal cells [22]. In mice, TZDs alter bone remodelling by suppressing bone formation and increasing bone resorption, resulting in decreased trabecular and cortical bone mass. Furthermore, TZD use in mice is associated with massive accumulation of adipocytes in the bone marrow cavity (reviewed in [48]). Recently, it has been shown that TZDs also affect osteocytes, the most abundant cells in bone, which orchestrate the bone remodelling process. In osteocytes, rosiglitazone increases production of proosteoblastic Wnt pathway inhibitors (sclerostin and dickkopf-related protein 1 [DKK1]) and of the proosteoclastic cytokine RANKL [17, 49].

In conclusion, the most common therapies, metformin and sulfonylurea, are safe for use in relation to their effect on bone, whereas less frequent therapies, such as TZDs and possibly SGLT2, may increase risk of fractures. Yet, with respect to bone disease in diabetes, the ideal glucose-lowering therapy would prevent fractures by supporting bone remodelling and, hence, increasing bone quality. There is no evidence that any existing glucose-lowering therapies provide such benefits to bone. However, new research offers a paradigm of glucoselowering drugs that may increase bone quality in individuals with diabetes by increasing bone turnover. In mice, a novel insulin sensitising agent, SR10171, which is an optimised derivative of 2-phenoxypropanoic acid and acts as an inverse agonist for PPAR $\gamma$ and a weak agonist for PPAR $\alpha$, increases bone mass and bone turnover by modulating the activity of osteoblasts, osteoclasts and osteocytes [17]. Thus, SR10171 may be considered a prototype drug that may target both insulin resistance and bone disease in diabetes.

\section{Perspectives and future directions}

In diabetes, bone disease has a complex pathology, hallmarked by increased fractures independent of BMD. This complexity results from the contribution of multiple physiological processes that are impaired in diabetes and contribute simultaneously to the negative effect of diabetes on bone and energy metabolism. There is an array of aspects of bone disease in diabetes that future studies must address, including the presence of biomarkers for the prediction of fracture risk and improvements in fracture healing, and multi-level pharmacological effects of glucose-lowering therapies on bone. There is also a need to develop a comprehensive animal model that would accurately reflect human bone disease in diabetes, including the intricacies of inter-organ communication.

Funding This work was supported by grants from NIH DK105825 and the American Diabetes Association ADA 7-13-BS-089.

Duality of interest The author declares that there is no duality of interest associated with this manuscript.

Contribution statement BLC was the sole contributor to this paper.

Open Access This article is distributed under the terms of the Creative Commons Attribution 4.0 International License (http:// creativecommons.org/licenses/by/4.0/), which permits unrestricted use, distribution, and reproduction in any medium, provided you give appropriate credit to the original author(s) and the source, provide a link to the Creative Commons license, and indicate if changes were made.

\section{References}

1. Schwartz AV (2017) Diabetes, bone and glucose-lowering agents: clinical outcomes. Diabetologia. doi: 10.1007/s00125-017-4283-6

2. Starup-Linde J, Frost M, Vestergaard P, Abrahamsen B (2017) Epidemiology of fractures in diabetes. Calcif Tissue Int 100:109121

3. Farr JN, Drake MT, Amin S, Melton LJ 3rd, McCready LK, Khosla S (2014) In vivo assessment of bone quality in postmenopausal women with type 2 diabetes. J Bone Miner Res 29:787-795

4. Lecka-Czernik B, Rosen CJ (2015) Energy excess, glucose utilization, and skeletal remodeling: new insights. J Bone Miner Res 30: 1356-1361

5. Krakauer JC, McKenna MJ, Buderer NF, Rao DS, Whitehouse FW, Parfitt AM (1995) Bone loss and bone turnover in diabetes. Diabetes 44:775-782

6. Rubin MR (2015) Bone cells and bone turnover in diabetes mellitus. Curr Osteoporos Rep 13:186-191

7. Patsch JM, Burghardt AJ, Yap SP et al (2013) Increased cortical porosity in type 2 diabetic postmenopausal women with fragility fractures. J Bone Miner Res 28:313-324

8. Nilsson AG, Sundh D, Johansson L et al (2016) Type 2 diabetes mellitus is associated with better bone microarchitecture but lower bone material strength and poorer physical function in elderly women: a population-based study. J Bone Miner Res. doi: $10.1002 / \mathrm{jbmr} .3057$

9. Wei J, Shimazu J, Makinistoglu MP et al (2015) Glucose uptake and Runx2 synergize to orchestrate osteoblast differentiation and bone formation. Cell 161:1576-1591

10. Clemens TL, Karsenty G (2011) The osteoblast: an insulin target cell controlling glucose homeostasis. J Bone Miner Res 26:677680

11. Ferron M, Wei J, Yoshizawa T et al (2010) Insulin signalling in osteoblasts integrates bone remodeling and energy metabolism. Cell 142:296-308

12. Kondegowda NG, Fenutria R, Pollack IR et al (2015) Osteoprotegerin and denosumab stimulate human beta cell proliferation through inhibition of the receptor activator of NF-kappaB ligand pathway. Cell Metab 22:77-85

13. Fulzele K, Riddle RC, Digirolamo DJ et al (2010) Insulin receptor signalling in osteoblasts regulates postnatal bone acquisition and body composition. Cell 142:309-319 
14. Wei J, Ferron M, Clarke CJ et al (2014) Bone-specific insulin resistance disrupts whole-body glucose homeostasis via decreased osteocalcin activation. J Clin Invest 124:1-13

15. Doucette CR, Horowitz MC, Berry R et al (2015) A high fat diet increases bone marrow adipose tissue (MAT) but does not alter trabecular or cortical bone mass in C57BL/6J mice. J Cell Physiol 230:2032-2037

16. Lecka-Czernik B, Stechschulte LA, Czernik PJ, Dowling AR (2015) High bone mass in adult mice with diet-induced obesity results from a combination of initial increase in bone mass followed by attenuation in bone formation; implications for high bone mass and decreased bone quality in obesity. Mol Cell Endocrinol 410: $35-41$

17. Stechschulte LA, Czernik PJ, Rotter ZC et al (2016) PPARG posttranslational modifications regulate bone formation and bone resorption. EBioMedicine 10:174-184

18. Lecka-Czernik B (2010) PPARs in bone: the role in bone cell differentiation and regulation of energy metabolism. Curr Osteoporos Rep 8:84-90

19. Ge C, Cawthorn WP, Li Y, Zhao G, MacDougald OA, Franceschi RT (2016) Reciprocal control of osteogenic and adipogenic differentiation by ERK/MAP kinase phosphorylation of Runx2 and PPARgamma transcription factors. J Cell Physiol 231:587-596

20. Stechschulte LA, Ge C, Hinds TD Jr, Sanchez ER, Franceschi RT, Lecka-Czernik B (2016) Protein phosphatase PP5 controls bone mass and the negative effects of rosiglitazone on bone through reciprocal regulation of PPARgamma (peroxisome proliferatoractivated receptor gamma) and RUNX2 (runt-related transcription factor 2). J Biol Chem 291:24475-24486

21. Wei W, Wang X, Yang M, Smith LC, Dechow PC, Wan Y (2010) PGC1beta mediates PPARgamma activation of osteoclastogenesis and rosiglitazone-induced bone loss. Cell Metab 11:503-516

22. Lazarenko OP, Rzonca SO, Hogue WR, Swain FL, Suva LJ, LeckaCzernik B (2007) Rosiglitazone induces decreases in bone mass and strength that are reminiscent of aged bone. Endocrinology 148:2669-2680

23. Abdallah BM, Ditzel N, Laborda J, Karsenty G, Kassem M (2015) DLK1 regulates whole-body glucose metabolism: a negative feedback regulation of the osteocalcin-insulin loop. Diabetes 64:30693080

24. Tevlin R, Seo EY, Marecic O et al (2017) Pharmacological rescue of diabetic skeletal stem cell niches. Sci Transl Med 9:eaag2809

25. Creecy A, Uppuganti S, Merkel AR et al (2016) Changes in the fracture resistance of bone with the progression of type 2 diabetes in the ZDSD rat. Calcif Tissue Int 99:289-301

26. Aikawa E, Fujita R, Asai M, Kaneda Y, Tamai K (2016) Receptor for advanced glycation end products-mediated signalling impairs the maintenance of bone marrow mesenchymal stromal cells in diabetic model mice. Stem Cells Dev 25:1721-1732

27. Ding KH, Wang ZZ, Hamrick MW et al (2006) Disordered osteoclast formation in RAGE-deficient mouse establishes an essential role for RAGE in diabetes related bone loss. Biochem Biophys Res Commun 340:1091-1097

28. Schwartz AV, Garnero P, Hillier TA et al (2009) Pentosidine and increased fracture risk in older adults with type 2 diabetes. J Clin Endocrinol Metab 94:2380-2386

29. Lafage-Proust MH, Roche B, Langer M et al (2015) Assessment of bone vascularization and its role in bone remodeling. Bonekey Rep $4: 662$

30. Weber DR, Haynes K, Leonard MB, Willi SM, Denburg MR (2015) Type 1 diabetes is associated with an increased risk of fracture across the life span: a population-based cohort study using the health improvement network (THIN). Diabetes Care 38:19131920
31. Shanbhogue VV, Hansen S, Frost M et al (2016) Compromised cortical bone compartment in type 2 diabetes mellitus patients with microvascular disease. Eur J Endocrinol 174:115-124

32. Tanikawa T, Okada Y, Tanikawa R, Tanaka Y (2009) Advanced glycation end products induce calcification of vascular smooth muscle cells through RAGE/p38 MAPK. J Vasc Res 46:572-580

33. Gohin S, Carriero A, Chenu C, Pitsillides AA, Arnett TR, Marenzana M (2016) The anabolic action of intermittent parathyroid hormone on cortical bone depends partly on its ability to induce nitric oxide-mediated vasorelaxation in BALB/c mice. Cell Biochem Funct 34:52-62

34. Eckardt K, Gorgens SW, Raschke S, Eckel J (2014) Myokines in insulin resistance and type 2 diabetes. Diabetologia 57:1087-1099

35. Yang X, Ricciardi BF, Hernandez-Soria A, Shi Y, Pleshko Camacho N, Bostrom MP (2007) Callus mineralization and maturation are delayed during fracture healing in interleukin- 6 knockout mice. Bone 41:928-936

36. Mera P, Laue K, Ferron $M$ et al (2016) Osteocalcin signalling in myofibers is necessary and sufficient for optimum adaptation to exercise. Cell Metab 23:1078-1092

37. Colaianni G, Cuscito C, Mongelli T et al (2015) The myokine irisin increases cortical bone mass. Proc Natl Acad Sci U S A 112:1215712162

38. Hampp C, Borders-Hemphill V, Moeny DG, Wysowski DK (2014) Use of antidiabetic drugs in the U.S., 2003-2012. Diabetes Care 37: 1367-1374

39. Jang WG, Kim EJ, Bae IH et al (2011) Metformin induces osteoblast differentiation via orphan nuclear receptor SHP-mediated transactivation of Runx2. Bone 48:885-893

40. Chen SC, Brooks R, Houskeeper J et al (2017) Metformin suppresses adipogenesis through both AMP-activated protein kinase (AMPK)-dependent and AMPK-independent mechanisms. Mol Cell Endocrinol 440:57-68

41. Zhou Z, Tang Y, Jin X et al (2016) Metformin inhibits advanced glycation end products-induced inflammatory response in murine macrophages partly through AMPK activation and RAGE/NFkappaB pathway suppression. J Diabetes Res 2016:4847812

42. Yu JW, Deng YP, Han X, Ren GF, Cai J, Jiang GJ (2016) Metformin improves the angiogenic functions of endothelial progenitor cells via activating AMPK/eNOS pathway in diabetic mice. Cardiovasc Diabetol 15:88

43. Mai QG, Zhang ZM, Xu S et al (2011) Metformin stimulates osteoprotegerin and reduces RANKL expression in osteoblasts and ovariectomized rats. J Cell Biochem 112:2902-2909

44. Ma P, Gu B, Xiong W et al (2014) Glimepiride promotes osteogenic differentiation in rat osteoblasts via the PI3K/Akt/eNOS pathway in a high glucose microenvironment. PLoS One 9:e112243

45. Fronczek-Sokol J, Pytlik M (2014) Effect of glimepiride on the skeletal system of ovariectomized and non-ovariectomized rats. Pharmacol Rep 66:412-417

46. Pereira M, Jeyabalan J, Jorgensen CS et al (2015) Chronic administration of glucagon-like peptide-1 receptor agonists improves trabecular bone mass and architecture in ovariectomised mice. Bone 81:459-467

47. Thrailkill KM, Nyman JS, Bunn RC et al (2016) The impact of SGLT2 inhibitors, compared with insulin, on diabetic bone disease in a mouse model of type 1 diabetes. Bone 82:101-107

48. Lecka-Czernik B (2010) Bone loss in diabetes: use of anti-diabetic thiazolidinediones and secondary osteoporosis. Curr Osteoporosis Rep 8:178-184

49. Mieczkowska A, Basle MF, Chappard D, Mabilleau G (2012) Thiazolidinediones induce osteocyte apoptosis by a $\mathrm{G}$ proteincoupled receptor 40-dependent mechanism. J Biol Chem 287: 23517-23526 